\title{
Deutschsein auf Probe
}

\section{Der Umgang deutsch-türkischer junger Erwachsener mit dem Optionsmodell}

\section{Von Claudia Diehl und Patrick Fick}

Zusammenfassung: In Deutschland müssen sich im Rahmen des „Optionsmodells“ junge Doppelstaater nach Erreichen der Volljährigkeit zwischen der deutschen Staatsbürgerschaft und der ihres Herkunftslandes entscheiden. Diese Entscheidung wird hier als Abwägungsergebnis rechtlich-pragmatischer und sozial-identifikativer Aspekte betrachtet. Die Auswertung einer standardisierten Befragung junger „Optionspflichtiger“ türkischer Herkunft, ergänzt um leitfadengestützte Interviews, zeigt zunächst, dass nahezu alle Personen beabsichtigen, den deutschen Pass zu behalten. Allerdings fällt diese Entscheidung nicht allen Befragten gleichermaßen leicht. Die Schwere der Entscheidung variiert vielmehr mit den wahrgenommenen Vor- und Nachteilen des jeweiligen Passes. So wird der deutsche Pass dann mit Leichtigkeit gewählt, wenn die Optionspflichtigen sich dadurch rechtliche Vorteile erhoffen und überzeugt sind, sich mit ihm als Deutsche fühlen zu können. Der Eindruck diskriminiert zu werden, eine hohe Bedeutung des türkischen Passes für die Familie sowie eine starke Identifikation mit der Türkei erschweren indes die Optionsentscheidung. Unabhängig davon hadern gerade die höher Gebildeten mit dem Optionszwang, möglicherweise ist dies ein Hinweis darauf, dass das Festhalten am Prinzip der Vermeidung von Mehrstaatigkeit besonders für gut integrierte Personen ein Einbürgerungshemmnis darstellt.

\section{Einleitung}

Im Jahr 2000 trat in Deutschland das sogenannte „Optionsmodell“ in Kraft. Es ist im Staatsangehörigkeitsgesetz (StAG) verankert und verknüpft die Einführung eines konditionalen jus soli („Bodenrecht“) mit dem Prinzip der Vermeidung von Mehrstaatigkeit. Das gegenwärtige deutsche Staatsbürgerschaftsrecht bricht damit einerseits mit der Tradition, die deutsche Staatsbürgerschaft allein auf Basis des jus sanguinis (,Blutrecht") zu vererben, bleibt ihr andererseits aber insofern treu, als die doppelte Staatsangehörigkeit weiterhin als zu vermeidendes „Übel” (BVerfG 21.5.1974) verstanden wird. Der deutsche Pass soll einen möglichst exklusiven Status darstellen, der eine eindeutige Grenze zwischen Staatsbürgern und NichtStaatsbürgern zieht.

Bemerkenswerterweise trifft dieses restriktive Moment ganz überwiegend Türkinnen und Türken, die größte in Deutschland lebende „Ausländergruppe“. Bei vielen anderen Nationalitätengruppen wird die doppelte Staatsbürgerschaft aus unterschiedlichen Gründen akzeptiert: Grundsätzlich bei Staatsbürgerinnen und -bürgern aus EU-Ländern oder der Schweiz, bei Personen, die aus Ländern stammen, in denen die Ausbürgerung nur schwer oder gar nicht möglich ist, oder bei anerkannten Flüchtlingen, denen die Ausbürgerung in der Regel nicht zugemutet wird. Während im Jahr 2011 lediglich 26\% aller Türkischstämmigen unter Hinnahme der doppelten Staatsbürgerschaft eingebürgert wurden, konnten rund $60 \%$ der Einwanderer aus Asien oder Afrika den deutschen Pass ohne Aufgabe ihrer jeweiligen ausländischen Staatsbürgerschaft annehmen (Statistisches Bundesamt 2012). Das proklamierte Prinzip der Vermeidung von Mehrstaatigkeit wird in der Realität also durchaus liberaler gehandhabt als es die öffentlichen Debatten erwarten lassen. Türkinnen und Türken sind allerdings weit überdurchschnittlich häufig von der Pflicht zur Aufgabe ihres Passes betroffen.

Für diese Gruppe ist das deutsche Optionsmodell, welches im Zentrum dieses Beitrags steht, daher besonders relevant. Es sieht vor, dass in Deutschland geborene Kinder von dauerhaft hier lebenden „Ausländern“ die doppelte Staatsbürgerschaft auf Zeit erhalten. Mit Eintritt der 
Volljährigkeit werden sie „optionspflichtig“ und müssen sich zwischen ihrer deutschen und ausländischen Staatsbürgerschaft entscheiden. Forschungspraktisch stellt die Optionsentscheidung eine besonders interessante Form der ansonsten schwer zu untersuchenden Einbürgerungsentscheidung dar. Die in Deutschland verhältnismäßig geringe Zahl an regulären Einbürgerungen könnte darauf zurückzuführen sein, dass diese in Ermangelung handfester rechtlicher Anreize häufig aufgeschoben wird und letztlich gar nicht stattfindet. Bei den jungen „Deutschen auf Probe“ besteht allerdings die Pflicht zur Wahl eines Passes. Das Optionsmodell forciert so diese Entscheidung als Übergang von zwei Mitgliedschaften zu einer exklusiven Staatsbürgerschaft innerhalb eines festgelegten - und damit „untersuchbaren“-Zeitrahmens.

Im Jahr 2008 erreichte die erste Kohorte Optionspflichtiger das kritische Alter. An dieser Gruppe lassen sich daher die der Einbürgerungsentscheidung zugrunde liegenden Beweggründe untersuchen, über die in vielerlei Hinsicht Unklarheit herrscht: Anhand existierender Datenquellen wie dem Mikrozensus können eingebürgerte und nicht-eingebürgerte Einwanderer und ihre Nachkommen zwar im Hinblick auf ihre sozialstrukturellen Merkmale verglichen werden (vgl. etwa Gresch / Kristen 2011), es mangelt jedoch an Informationen darüber, welche Merkmale und Beweggründe zum Zeitpunkt der Einbürgerung ausschlaggebend für diese Entscheidung waren. Letzteres ist mit Längsschnittdaten wie denen des Sozio-oekonomischen Panels zwar prinzipiell möglich, da aber im Zeitverlauf nur wenige Einwanderer eingebürgert werden, kann stellvertretend für die Einbürgerung meist nur die Einbürgerungs $a b$ sicht untersucht werden (vgl. Diehl / Blohm 2003, 2008; Hochman 2011). Eine Analyse der Entscheidungsprozesse der jungen Optionspflichtigen bietet zudem die Chance, die Folgen der rechtlich umstrittenen Optionsregelung genauer zu betrachten. Wie entscheidet sich die Mehrheit der Optionspflichtigen? Welchen Subgruppen fällt diese Entscheidung besonders leicht oder schwer? Welche Motive sind bei der Entscheidung für oder gegen den deutschen Pass ausschlaggebend?

Wir möchten im Folgenden diese Fragen beantworten und dazu die Ergebnisse einer empirischen Studie vorstellen, in deren Rahmen junge türkischstämmige „Optionspflichtige“ befragt wurden. In theoretischer Hinsicht geht der Beitrag von einem mehrdimensionalen Verständnis von Staatsbürgerschaft aus (vgl. Marshall 1950; Joppke 2010 a) und greift auf Arbeiten zurück, welche die Entscheidung für einen bestimmten Pass als Resultat einer Abwägung von sowohl rechtlich-pragmatischen als auch sozial-identifikativen Aspekten betrachten (Diehl / Blohm 2008, 2011). Ausgangspunkt ist dabei die Annahme, dass unter den spezifischen rechtlichen Rahmenbedingungen in Deutschland die Entscheidung für die deutsche Staatsbürgerschaft nicht allein mit dem Verweis auf die Gewährung von Rechten, die - wie das Wahlrecht oder der Zugang zur Beamtenlaufbahn - ausschließlich Staatsbürgern zur Verfügung stehen, erklärt werden kann. Vielmehr müssen die mit der Einbürgerung verbundenen sozialen und identifikativen Aspekte - etwa das Ausmaß der Identifikation mit dem Ziel- oder dem Herkunftsland (der Eltern) oder die soziale Integration - stärker in den Blick genommen werden.

Der Beitrag stellt zunächst kurz den rechtlichen Rahmen vor, innerhalb dessen Einbürgerungsentscheidungen in Deutschland getroffen werden; dabei wird ein Schwerpunkt auf die Darstellung des „Optionsmodells“ gelegt (2). Im Anschluss wird der bereits grob skizzierte theoretische Rahmen dargestellt, der auf der bisherigen Forschung zur Erklärung individueller und gruppenspezifischer Unterschiede im Einbürgerungsverhalten beruht (3). Daran anknüpfend werden das Design der Studie sowie die empirischen Befunde präsentiert (4). Die Untersuchung bietet Einblicke in das bislang wenig bekannte Entscheidungskalkül türkischstämmiger Optionspflichtiger (vgl. aber Weinmann / Becher / Babka von Gostomski 2012; Worbs / Scholz / Blicke 2012), ermöglicht eine erste Überprüfung der theoretischen Argumente bezüglich der Bestimmungsgründe von Einbürgerungsentscheidungen und kommt schließlich zu 
einer Abschätzung der Auswirkungen des Optionsmodells auf die Einbürgerungsprozesse der größten in Deutschland lebenden „Ausländergruppe“.

\section{Das deutsche Optionsmodell: Rechtliche Aspekte und quantitative Bedeutung}

Erst Anfang der 1990er Jahr wurde die relativ restriktive deutsche Einbürgerungspraxis, die auf das Reichs- und Staatsangehörigkeitsgesetz von 1913 zurückgeht, grundlegend reformiert. Dadurch erhielt ein großer Teil der längere Zeit in Deutschland lebenden Ausländer einen Regel- und bald darauf auch einen Rechtsanspruch auf Einbürgerung. Im Jahr 2000 trat begleitet von großer öffentlicher Aufmerksamkeit sowie scharfen politischen Auseinandersetzungen - eine Staatsbürgerschaftsreform in Kraft, welche das bisherige Recht um Elemente eines jus soli ergänzte (Wobbe / Otte 2000; Mertens 2004). An dem Prinzip der Vermeidung von Mehrstaatigkeit wurde, wie bereits erwähnt, festgehalten, allerdings wird seit der Neuregelung die doppelte Staatsbürgerschaft im Rahmen des „Optionsmodells“ zeitlich befristet akzeptiert. Diese Regelung ist weltweit ebenso einzigartig wie umstritten (Goldberg / Sen 1999; Weil 2001; Dornis 2002; Göbel-Zimmermann 2004; Gerdes / Faist 2006; von Münch 2007).

Entsprechend der neuen Praxis erhalten in Deutschland geborene Kinder ausländischer Eltern die deutsche Staatsangehörigkeit, sofern mindestens ein Elternteil das Daueraufenthaltsrecht besitzt und sich seit mindestens 8 Jahren in Deutschland aufhält ( 44 StAG). Neben der deutschen Staatsbürgerschaft darf das Kind zunächst auch die ausländische Staatsbürgerschaft behalten, die sich aus der Herkunft der Eltern ableitet. Bis zum 21. Lebensjahr besteht die Möglichkeit, einen Antrag auf Beibehaltung der doppelten Staatsbürgerschaft zu stellen, diesem wird aber in der Regel nur in den eingangs beschriebenen Ausnahmefällen stattgegeben. Möchten Optionspflichtige die deutsche Staatsbürgerschaft behalten, bleibt ihnen bis zum 23. Lebensjahr Zeit, den deutschen Behörden die entsprechenden Nachweise der Ausbürgerung vorzulegen. Reagieren sie bis zu diesem Zeitpunkt nicht, müssen sie damit rechnen, die deutsche Staatsbürgerschaft automatisch zu verlieren. Die erste Kohorte Optionspflichtiger wird im Jahr 2018 volljährig. Ab diesem Zeitpunkt werden sich dann jährlich etwa 40.000 „Deutsche auf Probe" für eine Staatsbürgerschaft entscheiden müssen.

Eine Übergangsregelung öffnete den Zugang zum Optionsmodell rückwirkend für Kinder, die zwischen 1990 und 2000 geboren wurden ( $\$ 40$ b StAG). Deren Eltern konnten den deutschen Pass für ihre Kinder beantragen, er wurde also nicht wie bei den „echten Optionskindern“ automatisch erworben. ${ }^{1}$ Von dieser Übergangsregelung haben rund 50.000 ausländische Kinder profitiert, gut $68 \%$ von ihnen haben türkische Wurzeln (Deutscher Bundestag 2008). Im Jahr 2008 wurde die erste Kohorte bestehend aus 3.300 Personen „optionspflichtig“, d.h. sie wurden von der zuständigen Staatsangehörigkeitsbehörde angeschrieben und aufgefordert, sich innerhalb von fünf Jahren, also bis zu ihrem 23. Geburtstag im Jahr 2013, für eine ihrer

1 Insgesamt wurde diese Regelung für weitaus weniger Kinder in Anspruch genommen als tatsächlich dazu berechtigt waren. Dies lag vermutlich daran, dass nicht alle Eltern über diese Regelung informiert waren. Im Gegensatz zu den ab 2000 geborenen Optionspflichtigen mussten die Antragsoptionspflichtigen zudem eine Gebühr von 500 DM entrichten. Ein Gesetzentwurf der damaligen Regierungskoalition, der sowohl eine Fristverlängerung um zwei Jahre als auch eine Absenkung der Einbürgerungsgebühr auf 100 DM vorsah, scheiterte am Votum des Bundesrates (Deutscher Bundesrat 2001: 206; siehe auch: Hagedorn 2001; Gerdes / Feist 2006). 
Staatsbürgerschaften zu entscheiden. ${ }^{2}$ Die Optionspflichtigen werden von den jeweiligen lokalen Staatsbürgerschaftsbehörden statistisch verwaltet, daher liegen bundesweit zentral erfasste Informationen über das Entscheidungsverhalten bislang nicht vor und sind auch zukünftig nicht zu erwarten. Abfragen bei einzelnen Behörden zeigen jedoch eine starke Tendenz zur Beibehaltung des deutschen Passes (Lämmermann 2011: $2 \mathrm{f}$ ). Die Daten weisen andererseits auch darauf hin, dass die Rückmeldequote seitens der Optionspflichtigen häufig unter 50 \% liegt. Die Gründe für diese Passivität können vielfältig und z.B. der zeit- und kostenaufwendigen Beschaffung der notwendigen Ausbürgerungsunterlagen geschuldet sein. Würde es bei dieser hohen Zahl an ,passiven“ Optionspflichtigen bleiben, könnten rund 5.000 Personen der Jahrgänge 1990 bis 1992 ihre deutsche Staatsbürgerschaft verlieren (Lämmermann 2011).

\section{Theoretischer Hintergrund: Rechtlich-pragmatische und sozial- identifikative Aspekte der Einbürgerung}

Die Frage, wie und aus welchen Beweggründen sich die Optionspflichtigen mehrheitlich entscheiden, führt zu der allgemeineren Frage, wie die Entscheidung für oder gegen den Pass des Ziellandes von den Einbürgerungsberechtigten getroffen wird. Diese stellen zwar die große Mehrheit der in Deutschland lebenden Einwanderer dar, dennoch lässt sich jedes Jahr nur eine kleine Minderheit von ihnen einbürgern (vgl. Statistisches Bundesamt 2012). Die traditionell niedrige und seit Jahren tendenziell sinkende Ausschöpfung dieses Einbürgerungspotenzials zeigt, dass die Einbürgerung keineswegs den natürlichen Endpunkt des Integrationsprozesses darstellt, wenngleich eine solche Abfolge als „deutsche Idealvorstellung“ der Einbürgerung betrachtet werden kann. Sie findet ihren Ausdruck etwa im folgenden Zitat eines parlamentarischen Staatssekretärs beim Bundesminister des Innern: „Für uns ist die Einbürgerung Ausdruck gelungener Integration. Sie steht nicht am Anfang, sondern sie setzt bereits eine Reihe von Integrationsleistungen voraus.“ (Deutscher Bundestag 2011: 16469)

Die seit Jahrzehnten relativ geringen Einbürgerungsquoten von „Ausländern“ in Deutschland könnten dementsprechend als Zeichen einer gescheiterten Integration oder gar der Abkehr von der deutschen Gesellschaft interpretiert werden. In diese Richtung zielt das Argument von Schiffauer $(2007,2008)$, wenngleich er die Ursache für die gescheiterte Integration stärker auf Seiten der Mehrheitsgesellschaft verortet. Die geringe Neigung zur Annahme der deutschen Staatsbürgerschaft interpretiert er als (Abwehr-)Reaktion der Migranten - insbesondere der Muslime in der Folge islamkritischer Debatten nach 9/11 - auf einen wahrgenommenen Mangel an Akzeptanz. Ein Blick auf die nationalitätenspezifische Varianz der Einbürgerungsraten zeigt allerdings, dass gerade Türkinnen und Türken eine höhere Bereitschaft zur Einbürgerung aufweisen als die Angehörigen anderer Nationalitäten (vgl. Diehl / Blohm 2008; Statistisches Bundesamt 2012) - und dies, obwohl gerade ihnen die Möglichkeit zur Beibehaltung des türkischen Passes fehlt.

Eine theoriegeleitete Erklärung von Einbürgerungsentscheidungen muss nicht nur den individuellen, sondern auch den skizzierten nationalitätenspezifischen Unterschieden im Einbürgerungsverhalten Rechnung tragen können. Dabei gilt es, unterschiedliche Aspekte der Entscheidung zu berücksichtigen. Joppke (2010 a) differenziert drei Dimensionen von Staatsbürgerschaft: Status, Recht und Identität. Die Unterscheidung dient vor allem zur Betrachtung unterschiedlicher Konfigurationen von Staatsbürgerschaft im Zeitverlauf oder zwischen Staa-

2 Die in der Regel kommunale Organisation der Optionspflicht führt dazu, dass die Regelung mit unterschiedlicher Intensität umgesetzt wird, etwa indem Betroffene lediglich einmalig oder wiederholt angeschrieben werden. In Rheinland-Pfalz wird die Aufforderung zur Optionsentscheidung z.B. zusammen mit einem Schreiben des Ministerpräsidenten versandt, der für die Beibehaltung der deutschen Staatsbürgerschaft wirbt. 
ten. ${ }^{3}$ Eine solche Differenzierung verschiedener Bedeutungsebenen von Staatsbürgerschaft ist aber nicht nur auf der Makro-, sondern auch auf der Mikroebene von Bedeutung; dies gilt insbesondere für die beiden letztgenannten Aspekte. In diesem Zusammenhang haben Diehl und Blohm (2003) versucht, die empirische Bedeutung rechtlich-pragmatischer sowie sozialidentifikativer Einbürgerungsanreize zu untersuchen, um so individuelle und nationalitätenspezifische Unterschiede im Einbürgerungsverhalten zu erklären.

Rechtlich-pragmatische Einbürgerungsanreize ergeben sich aus der Differenz der universellen staatsbürgerlichen Rechte und den Rechten von Nicht-Staatsbürgern, also Ausländern. Ist diese Differenz unbedeutend, bestehen nur geringe rechtlich-pragmatische Einbürgerungsanreize, ist sie ausgeprägt, können rechtliche Aspekte bei der Einbürgerung eine entscheidende Rolle spielen. Dies lässt sich besonders gut am Beispiel klassischer Einwanderungsländer wie den USA, Kanada oder Australien verdeutlichen, wo diese Anreize einen wichtigen Beitrag zur Erklärung von Intra- und Intergruppenunterschieden im Einbürgerungsverhalten leisten (Barkan / Khokhlov 1980; Portes / Mozo 1985; Alvarez 1987; Portes / Curtis 1987; Evans 1988; Yang 1994 a, 1994 b; Portes / Rumbaut 1996; Aguirre / Saenz 2002; Van Hook / Brown / Bean 2006). In den USA wurde beispielsweise Mitte der 1990er Jahre die Zahlung von Transferleistungen an Legal Permanent Residents eingeschränkt und ihre sozialen Rechte deutlich beschnitten (Yang 1994 b; Schuck 1998; Borjas 2001; Bloemraad 2006). Weitere Anreize sind ein erleichterter Familiennachzug für Angehörige von Staatsbürgern (Jasso / Rosenzweig 1986) oder das Recht zur Teilnahme an Wahlen und Abstimmungen (Plascencia / Freeman / Setzler 2003).

Der sich jeweils ergebende Nutzen der Einbürgerung ist individuell variabel. So ist ein für Eingebürgerte problemloserer Zugang zum Arbeitsmarkt oder zu bestimmten Arbeitsmarktsegmenten in der Regel für höher Gebildete von größerer Bedeutung als für einfache Arbeiter (klassisch: Bernard 1936; Portes / Mozo 1985; für bestätigende Befunde: Jasso / Rosenzweig 1986; Portes / Curtis 1987; kritisch: Evans 1988; Liang 1994; für Einbürgerungsabsicht: Massey / Akresh 2006; Euwals et al. 2010). Auch die wahrgenommenen Vorteile breiterer Partizipationsmöglichkeiten variieren mit dem individuellen politischen Interesse sowie mit der Fähigkeit und Kompetenz zur Nutzung dieser Rechte. So gibt es etwa Hinweise auf einen eigenständigen positiven Einfluss des politischen Interesses auf die Einbürgerungsneigung (Freeman et al. 2002; Pantoja / Gershon 2006).

In Deutschland scheint die rechtlich-pragmatische Nutzendimension demgegenüber insgesamt eher schwach, nationalitätenspezifisch jedoch durchaus unterschiedlich stark ausgeprägt zu sein. Hat man den Status des legal und dauerhaft im Land lebenden „Ausländers“ - des denizen (vgl. Hammar 1990) - erreicht, ist die Differenz zum Status des citizen relativ gering. So ist der Zugang zum Arbeitsmarkt weitgehend frei und lediglich für bestimmte Bereiche beschränkt, etwa für akademische Heilberufe oder die Beamtenlaufbahn. Dies spiegelt die Gleichzeitigkeit eines relativ restriktiven Staatsbürgerschaftsrechts und der Anwesenheit einer großen Zahl dauerhaft in Deutschland lebender (und in Teilen hier geborener) Ausländer wider, denen unterhalb der Ebene der Staatsbürgerschaft viele soziale, zivile und politische Rechte eingeräumt wurden (Hammar 1990; Castles 1994; Santel 1998; Prümm 2004; Nassehi 2010).

Seit der Einführung der Unionsbürgerschaft im Jahr 1992 genießen EU-Angehörige sogar die nahezu vollständige rechtliche Gleichstellung mit Deutschen (zur Diskussion der EUStaatsbürgerschaft als Staatsbürgerschaft ,light“ siehe Joppke 2010 b) und dürfen zumindest

3 So können in Bezug auf den Status die Modi des Erwerbs einer Staatsbürgerschaft (jus soli vs. jus sanguinis) sowie deren Exklusivität (Vermeidung doppelter Staatsbürgerschaft) unterschieden werden, auf der Rechte-Dimension der Umfang sowie der Grad der Universalität bzw. Partikularität staatsbürgerlicher Rechte und hinsichtlich der Identität etwa der Grad der Betonung einer nationalen Identität durch den Staat bzw. durch seine Akteure (Joppke 2007). 
an Europa- und Kommunalwahlen teilnehmen. In Deutschland lebende Drittstaatenangehörige sind indes vom aktiven und passiven Wahlrecht ausgeschlossen; zudem ergeben sich für eingebürgerte Drittstaatenangehörige, die Sozialleistungen beziehen, Vorteile beim Familiennachzug. Darüber hinaus ermöglicht ein deutscher Pass das freie Reise- und Aufenthaltsrecht innerhalb der EU sowie visumfreie oder erleichterte Reisen in Staaten außerhalb der EU. Die höhere Einbürgerungsneigung von Nicht-EU-Angehörigen kann also durchaus auf die für diese Gruppe höheren rechtlich-pragmatischen Einbürgerungsanreize zurückzuführen sein.

Allerdings zeigen nationalitätenspezifische Unterschiede im Einbürgerungsverhalten von Nicht-EU-Ausländern, dass eine Erklärung, die ausschließlich rechtliche Einbürgerungsanreize berücksichtigt, unzulänglich ist. Daher betonen Diehl und Blohm $(2003,2008)$ die $B e-$ deutung sozialer und identifikativer Anreize der Einbürgerung. Sie beschreiben Einbürgerung als eine „Zugehörigkeitsentscheidung“, bei der neben den rechtlichen Vor- und Nachteilen die sozialen und identifikativen Implikationen dieses Schrittes eine wichtige Rolle spielen. Einbürgerung stellt in dieser Perspektive eine Form des individual ethnic boundary crossing dar, bei dem Minderheitenangehörige die Merkmale der Mehrheit annehmen und so individuell eine salient bleibende ethnische Grenze überschreiten (vgl. Alba 2005; Wimmer 2008; kritisch dazu: Hochman 2011). Die Einbürgerung kann insofern Teil einer solchen Strategie sein, als sie die Möglichkeit bietet, die subjektiv wahrgenommene Zugehörigkeit zur - statushöheren - Mehrheit auch formal zu besiegeln. Diese „Neupositionierung“ steht jedoch nur den Individuen offen, die bereits einen gewissen Zugang zur Mehrheitsgesellschaft, etwa in Form freundschaftlicher Kontakte, besitzen. Fehlt dieser Zugang, droht das boundary crossing zu scheitern und in der Marginalisierung zu enden, besonders dann, wenn die Aufgabe des alten Passes von der Herkunftsgruppe abgelehnt wird. Es kann also davon ausgegangen werden, dass fehlende Akzeptanz durch und Kontakte zu Mehrheitsangehörigen, die einen etwaigen Entzug der sozialen Anerkennung in der eigenen ethnischen Gruppe abfedern können, und / oder die Ablehnung dieses Schrittes seitens der Familie die Einbürgerungsentscheidung unabhängig von den rechtlichen Implikationen erschweren.

Die skizzierten Anreize der Einbürgerung lassen eine systematische individuelle Varianz in der Optionsentscheidung erwarten. So dürfte die Einbürgerung für Personen mit hoher Bildung und einem ausgeprägten Interesse an deutscher Politik besonders attraktiv sein, d.h. einen hohen rechtlich-pragmatischen Nutzen besitzen, eröffnet sie doch Zugang zu unkomplizierten Auslandsreisen, zum Beamtenstatus und zum Wahlrecht. Hohe identifikative und soziale Einbürgerungsanreize dürften dann bestehen, wenn eine Optionspflichtige sich ohnehin schon stark als Deutsche fühlt, Kontakte zu Mehrheitsangehörigen besitzt, sich von diesen anerkannt und wenig diskriminiert fühlt und wenn Freunde und Familienmitglieder die Einbürgerung akzeptieren. Ausgehend von diesen Annahmen soll im Folgenden das Einbürgerungsverhalten der Optionspflichtigen genauer analysiert werden.

\section{Deutsche/r bleiben? - Das Entscheidungsverhalten türkischstämmiger Optionspflichtiger}

Die nachfolgend dargestellten Befunde beruhen auf der Studie Deutsche/r bleiben?, die im Sommer 2011 an der Georg-August-Universität Göttingen durchgeführt wurde. Auf der Grundlage einer registerbasierten Zufallsstichprobe wurden die Adressen von türkischstämmigen Optionspflichtigen der Jahrgänge 1990 bis 1993 ermittelt. ${ }^{4}$ Die Ausgangsstichprobe

4 Dazu wurde auf die Melderegister der Städte Augsburg, Bielefeld, Bremen, Bremerhaven, Dortmund, Düsseldorf und Hannover zurückgegriffen. 
wurde außerdem mit Geodaten der Firma microm ${ }^{5}$ angereichert. Dadurch standen auf Aggregatebene Informationen über die soziale Herkunft aller angeschriebenen Personen zur Verfügung, so dass zumindest ansatzweise die Stichprobenqualität untersucht werden konnte (vgl. Fick / Diehl 2013). Als Indikatoren herangezogen wurden der Kaufkraftindex, der die durchschnittliche Kaufkraft des Straßenabschnitts abbildet, in dem der oder die Befragte wohnt, ${ }^{6}$ sowie der Ausländerindex. Letzterer lag als ordinale Variable vor (von 0 „,niedrigster Anteil“ bis 8 ,höchster Anteil“"). Auf Straßenabschnittsebene lässt die Variable eine Einschätzung über die Zusammensetzung der Nachbarschaft des Haushaltes zu, dem der oder die jeweilige Befragte angehört.

Tabelle 1: Ausschöpfungsquote und Stichprobenqualität

\begin{tabular}{|c|c|c|}
\hline Bruttostichprobe $(N=500)$ & & \\
\hline $\begin{array}{l}\text { Anteil „stichprobenneutraler“ Ausfälle } \\
\text { Ausschöpfungsquote }^{1}\end{array}$ & $\begin{array}{r}3,4 \\
55,5\end{array}$ & $\begin{array}{l}(17) \\
(268)\end{array}$ \\
\hline $\begin{array}{l}\text { Bereinigte Bruttostichprobe }(N=483) \text { : } \\
\text { Mittelwertdifferenzen (Teilnehmer }-V\end{array}$ & & \\
\hline $\begin{array}{l}\text { Frauenanteil }{ }^{2} \\
\text { Ausländerindex }\left(\text { Min: } 0 ; \text { Max: }^{3}\right)^{3} \\
\text { Kaufkraftindex }(B R D=100)^{3}\end{array}$ & $\begin{array}{l}+7,9^{*} \\
+0,1 \\
+0,3\end{array}$ & \\
\hline
\end{tabular}

${ }^{1}$ Ausschöpfungsquote=Interviews/(versandte Fragebögen - Ausfälle), entspricht RR5 der AAPOR (2011)

Standard-Definition

${ }^{2}$ Chi-Quadrat-Test

${ }^{3}$ zweiseitiger t-Test

$* \mathrm{p}<, 10$

Quelle: Deutsche/r bleiben?

Tabelle 1 zeigt, dass insgesamt 268 Personen an der schriftlichen Befragung teilnahmen; die Ausschöpfungsquote ist mit $56 \%$ relativ hoch. ${ }^{7}$ Daneben ermöglichen die zugespielten microm-Indikatoren einen Vergleich der Befragten mit nicht-teilnehmenden Zielpersonen und erlauben somit einen Einblick in die Qualität der gewonnenen Stichprobe: Zwar zeigt der Abgleich mit den Melderegisterdaten, dass überdurchschnittlich viele Frauen an der Studie teilgenommen haben, eine Verzerrung entlang sozialer (Kaufkraftindex) oder integrationsbezogener (Ausländerindex) Indikatoren scheint jedoch nicht vorzuliegen. Die Stichprobe ist somit zwar relativ klein, im Hinblick auf die hier untersuchten Merkmale aber wenig selektiv.

Das Erhebungsinstrument, ein standardisierter Fragebogen von ca. 15 Minuten Länge, enthielt Fragen zu Optionsverhalten bzw. -absicht, Items zum soziodemografischen Hintergrund und Indikatoren zur Messung der rechtlich-pragmatischen (politisches Interesse, Bildung) und sozial-identifikativen Einbürgerungsanreize (ethnische Identifikation, subjektive Diskriminierungserfahrung, Einstellungen von Familie, Freunden und Partnerin bzw. Partner zur Einbürgerung). Mit acht Befragten, denen die Optionsentscheidung unterschiedlich schwer bzw. leicht fiel und deren ethnische Selbstidentifizierung variierte, wurden zusätzlich qualitative Interviews von bis zu einer Stunde Länge geführt; auf die dabei gewonnenen Informationen wird im Folgenden an einzelnen Stellen zur Illustration zurückgegriffen.

5 Weitere Informationen über die hauptsächlich im Bereich des Konsumentenmarketings verwendeten Geodaten unter www.microm-online.de. Mit einem ähnlichen Vorgehen wurde der spezielle MICROM-SOEP-Datensatz erstellt (Goebel et al. 2007).

6 Der microm Bundesdurchschnitt ist dabei auf den Wert 100 festgesetzt, darunter liegende Werte stehen somit für eine unterdurchschnittliche Kaufkraft des Straßenabschnittes des oder der Befragten.

7 Die Ausschöpfungsquote entspricht RR5 der AAPOR (2011) Standard-Definition. 
Der folgende Abschnitt beschreibt die Optionspflichtigen zunächst anhand ausgewählter Hintergrundvariablen (4.1) und stellt ihre (beabsichtigte) Optionsentscheidung dar (4.2). Darauf aufbauend wird auf die Bedeutung rechtlich-pragmatischer und sozial-identifikativer Entscheidungsaspekte eingegangen (4.3).

\subsection{Türkischstämmige Optionspflichtige - Ein Porträt}

Aus dem deskriptiven Überblick in Tabelle 2 geht zunächst das relativ hohe Bildungsniveau der befragten türkischstämmigen Optionspflichtigen hervor. Ein Vergleich der entsprechenden Altersgruppe der im Mikrozensus befragten Türkinnen und Türken sowie der Deutschen ohne Migrationshintergrund bestätigt, dass die türkischen Optionspflichtigen eine relativ gut gebildete Subgruppe darstellen. Knapp $48 \%$ gaben an, die mittlere Reife erreicht zu haben und $27 \%$ besitzen das Abitur, folglich ist hier auch der Abstand zu den Deutschen ohne Migrationshintergrund gering. Für diese liegen die entsprechenden Vergleichswerte im Mikrozensus bei $44 \%$ (mittlere Reife) bzw. $30 \%$ (Abitur) während nur 32\% der im Mikrozensus befragten Türkinnen und Türken die mittlere Reife und $12 \%$ das Abitur erreicht haben. $63 \%$ der Optionspflichtigen schätzen ihre Deutschkenntnisse als sehr gut ein (ohne Darstellung). Zugleich ist Deutsch die dominierende Sprache. Mehr als die Hälfte gibt an, immer Deutsch mit Freundinnen und Freunden zu sprechen. Bei der Kommunikation mit der Familie wird seltener auf Deutsch zurückgegriffen, doch auch hier geben rund $57 \%$ an, immer oder oft mit der Familie Deutsch zu sprechen. Dieser Befund einer fortgeschrittenen strukturellen und kognitiven Integration der Optionspflichtigen ist bei der Interpretation der weiteren Ergebnisse zu berücksichtigen: Wie oben dargestellt, musste für die hier betrachteten Personen das Optionsmodell von den Eltern eigens beantragt werden, und dies scheint zumindest in Bezug auf das Bildungsniveau eine positive Selektion dieser Gruppe bewirkt zu haben. 
Tabelle 2: Ausgewählte Merkmale der Optionspflichtigen (in \%)

\begin{tabular}{|c|c|c|}
\hline \multicolumn{3}{|l|}{ Geschlecht (268) } \\
\hline weiblich & 51,1 & \\
\hline \multicolumn{3}{|l|}{ Bildungsniveau (266) } \\
\hline (Noch) Keinen Abschluss & 8,7 & \\
\hline Hauptschulabschluss & 16,2 & \\
\hline Mittlere Reife & 47,7 & \\
\hline Abitur & 27,4 & \\
\hline Deutsch sprechen... & ...mit der Familie (268) & ...mit Freunden (268) \\
\hline Immer & 7,6 & 52,2 \\
\hline Oft & 49,8 & 42,2 \\
\hline Manchmal & 27,9 & 4,5 \\
\hline Selten & 10,9 & 0,8 \\
\hline $\mathrm{Nie}$ & 3,8 & 0,4 \\
\hline Interesse für... & ...deutsche Politik (265) & ...türkische Politik (265) \\
\hline Sehr stark & 10,6 & 13,6 \\
\hline Stark & 20,8 & 16,6 \\
\hline Mittel & 41,9 & 29,1 \\
\hline Wenig & 16,6 & 19,3 \\
\hline Überhaupt nicht & 10,2 & 21,5 \\
\hline \multirow[t]{2}{*}{ Anteil der max. drei Freunde... } & ...mit türkischen Wurzeln (259) & $\begin{array}{l}\text {...der zur türkischen Staatsbür- } \\
\text { gerschaft rät (260) }\end{array}$ \\
\hline & 66,0 & 10,6 \\
\hline Benannte Freunde ausschließlich... & ...türkische Wurzeln (259) & $\begin{array}{l}\text {...nicht-türkischer Herkunft } \\
\text { (259) }\end{array}$ \\
\hline $\mathrm{Ja}$ & 43,2 & 11,6 \\
\hline Gefühl als... & ...Deutsche/r (264) & ...Türke / in (268) \\
\hline Voll und ganz & 11,0 & 37,3 \\
\hline Überwiegend & 17,1 & 31,3 \\
\hline In mancher Beziehung & 38,6 & 21,6 \\
\hline Kaum & 19,7 & 5,2 \\
\hline Gar nicht & 13,6 & 4,5 \\
\hline Wie oft in Deutschland... & $\begin{array}{l}\text {...werden Türken schlechter } \\
\text { behandelt (266) }\end{array}$ & $\begin{array}{l}\text {...wurden Sie schlechter behan- } \\
\text { delt? (265) }\end{array}$ \\
\hline Sehr oft & 20,7 & 9,1 \\
\hline Oft & 36,5 & 19,3 \\
\hline Manchmal & 27,4 & 24,9 \\
\hline Seltener & 11,3 & 23,4 \\
\hline Nie & 4,1 & 23,4 \\
\hline Rat zur türk. Staatsbürgerschaft von... & ...Vater (265) & ...Mutter (264) \\
\hline $\mathrm{Ja}$ & 10,9 & 11,4 \\
\hline \multicolumn{3}{|l|}{ Später in Türkei leben (268) } \\
\hline Für immer & 16,8 & \\
\hline Für einige Jahre & 35,5 & \\
\hline Nein & 47,8 & \\
\hline
\end{tabular}

Quelle: Deutsche/r bleiben? 
Das individuelle Interesse für die deutsche oder türkische Politik ist als Information über den Grad der Verbundenheit mit der jeweiligen Gesellschaft interessant. Zugleich dürfte ein starkes politisches Interesse für die Staatsangehörigkeitsentscheidung relevant sein, weil aktives wie passives Wahlrecht von ihr abhängen. Bezüglich des Interesses an der Politik der beiden Länder gibt es keine bedeutenden Unterschiede. Von den befragten - in Deutschland geborenen und aufgewachsenen - jungen Erwachsenen interessieren sich jeweils ca. $30 \%$ für die deutsche bzw. die türkische Politik sehr stark oder stark.

Die fortgeschrittene strukturelle Integration der Optionspflichtigen geht offenbar nicht mit einer Distanz zur eigenen ethnischen Gruppe einher. In der Befragung konnten Angaben zu maximal drei engen Freunden gemacht werden. Der Anteil der türkischstämmigen Freunde mit direkter oder indirekter Migrationserfahrung liegt bei $66 \%$, $43 \%$ der Befragten nennen hier sogar ausschließlich Personen mit türkischen Wurzeln. Die Angaben bezüglich des Zugehörigkeitsgefühls weisen in eine ähnliche Richtung. $69 \%$ der Befragten identifizieren sich voll und ganz bzw. überwiegend als Türkin bzw. Türke, nur rund $28 \%$ geben an, sich voll und ganz oder überwiegend als Deutsche bzw. Deutscher zu fühlen. Eine (hier nicht dargestellte) Kreuztabellierung zeigt, dass mit steigendem Zugehörigkeitsgefühl zur Türkei die Identifikation mit Deutschland sinkt, lediglich $13 \%$ weisen eine „hybride Identität“ auf und fühlen sich voll und ganz oder überwiegend beiden Gesellschaften zugehörig. Ein Vergleich mit dem Sozio-oekonomischen Panel (SOEP), der aufgrund der geringen Fallzahl in Deutschland geborener Personen türkischer Nationalität im Alter der Optionspflichtigen mit Bedacht zu interpretieren ist, zeigt, dass sich die strukturell weitgehend assimilierten Optionspflichtigen in unserer Stichprobe nur unwesentlich häufiger als „,deutsch“ identifizieren als die entsprechend im SOEP befragten Türken (SOEP: $9 \%$,voll und ganz“, 39\% ,in mancher Beziehung“, $9 \%$ „gar nicht").

Tabelle 2 informiert auch über die subjektive Wahrnehmung von Diskriminierung. Vor dem Hintergrund des ethnic boundary crossing Paradigmas kann dieser Indikator Auskunft über die empfundene Stärke der ethnischen Grenzziehung auf der Seite der Optionspflichtigen geben. Mit steigender Diskriminierungswahrnehmung könnte die Erwartung abnehmen, trotz eines deutschen Passes und zunehmender „Integrationsleistung“ auf Akzeptanz bzw. NichtDiskriminierung in der Aufnahmegesellschaft zu stoßen. $57 \%$ der Befragten sind der Ansicht, dass Türkinnen und Türken in Deutschland oft oder sehr oft wegen ihrer Herkunft schlechter behandelt werden, allerdings gibt ein weitaus geringerer Anteil von $28 \%$ an, sehr oft oder oft persönlich von einer solchen schlechteren Behandlung betroffen gewesen zu sein.

Der deutschen Staatsbürgerschaft stehen sowohl die Eltern als auch die Freundinnen und Freunde der Befragten generell sehr positiv gegenüber. Dies überrascht insbesondere im Falle der Eltern nicht, schließlich waren sie es, die das Optionsmodell und damit den deutschen Pass für ihre Kinder beantragt haben - trotz der nicht unwesentlichen Kosten von 500 DM für die Antragsstellung im Jahr 2000. Erstaunlich ist vor diesem Hintergrund vielmehr, dass jeweils ca. $11 \%$ der Mütter bzw. Väter ihren Kindern dazu raten bzw. geraten haben, den türkischen Pass zu behalten. Möglicherweise sind diese Eltern im Laufe der Jahre skeptischer geworden, was die Aufgabe der türkischen Staatsbürgerschaft angeht - oder es bestand von Anfang an Uneinigkeit innerhalb der Familie. Dessen ungeachtet verfügt die Mehrheit der Optionspflichtigen über ein direktes soziales Umfeld, welches der Beibehaltung des deutschen unter Aufgabe des türkischen Passes zumindest nicht ablehnend gegenübersteht, was auch für die drei genannten engen und meist türkischstämmigen Freunde gilt, von denen lediglich $11 \%$ zur Beibehaltung des türkischen Passes raten.

Schließlich wurden die in Deutschland geborenen und aufgewachsenen Optionspflichtigen nach ihren Zukunftsperspektiven befragt, demzufolge können sich 17 \% vorstellen, für immer 
in der Türkei zu leben, $36 \%$ immerhin für einige Jahre, die übrigen ziehen beides nicht in Betracht.

\subsection{Die Optionsentscheidung-Deutsche/r bleiben?}

In Tabelle 3 sind die Informationen zum Optionsverhalten bzw. zur Optionsabsicht der türkischstämmigen Optionspflichtigen veranschaulicht. Von denjenigen, die bereits auf das Schreiben der Staatsbürgerschaftsbehörde reagiert haben, hat niemand für die Beibehaltung der türkischen Staatsbürgerschaft votiert, gut $5 \%$ haben die doppelte Staatsbürgerschaft beantragt; uns ist allerdings nicht bekannt, ob über diesen Antrag bereits entschieden wurde. Diejenigen, die bislang noch keine Entscheidung getroffen haben, wurden zu ihrer Entscheidungstendenz befragt. Lediglich drei Personen ( $2 \%$ ) gaben dabei an, eher den türkischen Pass behalten zu wollen, rund $12 \%$ sind noch unentschieden. Eingangs wurde gezeigt, dass die Entscheidung gegen den deutschen und für den türkischen Pass offenbar meist in der Form fällt, dass die behördliche Aufforderung zur Entscheidung ignoriert wird und infolgedessen der Entzug des deutschen Passes bei Erreichen des 23. Lebensjahres droht. Es ist daher durchaus denkbar, dass es bei den unentschiedenen Optionspflichtigen letztlich doch zur Beibehaltung des türkischen Passes und damit zum Entzug der deutschen Staatsbürgerschaft kommt. Zudem sei an dieser Stelle nochmals daraufhin gewiesen, dass sich unter den ,echten Optionspflichtigen" - also den Geburtskohorten ab dem Jahr 2000, die automatisch unter die Optionsregelung fallen und nicht auf ausdrücklichen Wunsch und Antrag ihrer Eltern den deutschen Pass qua Geburt in Deutschland erhalten - voraussichtlich deutlich mehr Personen befinden werden, die beispielsweise ein weniger unterstützendes familiäres Umfeld besitzen oder aus anderen Gründen eher bereit sind, ihren deutschen Pass aufzugeben. 
Tabelle 3: Optionsentscheidung und Schwere bzw. Sicherheit der Entscheidung (in \%)

\begin{tabular}{|c|c|c|}
\hline Bereits entschieden (131) & & \\
\hline Insgesamt & 48,9 & \\
\hline \multicolumn{3}{|l|}{ davon... } \\
\hline ...für die deutsche Staatsbürgerschaft & & 94,7 \\
\hline ...für türkische Staatsbürgerschaft & & 0,0 \\
\hline ...Antrag auf doppelte Staatsbürgerschaft & & 5,3 \\
\hline \multicolumn{3}{|l|}{ Entscheidung noch ausstehend (144) } \\
\hline Insgesamt & 51,1 & \\
\hline \multicolumn{3}{|l|}{ davon... } \\
\hline ...eher für die deutsche Staatsbürgerschaft & & 86,1 \\
\hline ...eher für die türkische Staatsbürgerschaft & & 2,2 \\
\hline ...unentschlossen & & 11,7 \\
\hline \multicolumn{3}{|l|}{ Optionsentscheidung fällt / fiel... (262) } \\
\hline ...überhaupt nicht schwer & 51,2 & \\
\hline ...weniger schwer & 30,9 & \\
\hline ...ziemlich schwer & 9,9 & \\
\hline ...sehr schwer & 8,0 & \\
\hline \multicolumn{3}{|c|}{ Befragte/r ist / war sich bei der Optionsentscheidung... (259) } \\
\hline ...ganz sicher & 61,0 & \\
\hline ...ziemlich sicher & 28,6 & \\
\hline ...nicht so sicher & 6,6 & \\
\hline ...gar nicht sicher & 3,9 & \\
\hline
\end{tabular}

Quelle: Deutsche/r bleiben?

Unabhängig davon, ob sich die im Rahmen unserer Studie befragten Optionspflichtigen bereits für einen Pass entschieden haben oder ob diese Entscheidung noch aussteht, wurden sie danach gefragt, mit welcher Sicherheit sie ihre Entscheidung trafen bzw. treffen werden und wie schwer ihnen die Wahl zwischen den beiden Pässen fiel bzw. fällt. Dabei zeigt sich, dass etwa der Hälfte die Entscheidung überhaupt nicht schwer fällt, knapp einem Fünftel fällt sie allerdings ziemlich oder sogar sehr schwer. Mehr als $60 \%$ sind sich ihrer Entscheidung ganz sicher, der Rest ist sich überwiegend ,ziemlich sicher“ (vgl. Tabelle 3).

Damit verlagert sich das Erklärungsproblem auf die Frage, welche Faktoren es sind, die die Staatsbürgerschaftsentscheidung zu einer Entscheidung werden lassen, die schwer fällt und / oder mit Unsicherheiten behaftet ist. Erhöht eine wahrgenommene hohe instrumentelle Bedeutung des deutschen Passes die Leichtigkeit, mit der die Entscheidung gefällt wird? Spielt das individuelle Zugehörigkeitsgefühl zu Deutschland oder der Türkei eine Rolle? Fällt es zum Beispiel denjenigen, die sich als Deutsche fühlen, leichter, den türkischen Pass aufzugeben? Erschweren Diskriminierungserfahrungen die Entscheidung, weil es ohnehin aussichtslos erscheint, jemals als Deutsche/r akzeptiert zu werden? Es stellt sich außerdem die Frage, welche Rolle die Einstellungen wichtiger Bezugspersonen wie Eltern oder Freunde bei der Entscheidung über die Beibehaltung des deutschen und die Aufgabe des türkischen Passes spielen. 


\subsection{Die Bedeutung rechtlich-pragmatischer und sozial-identifikativer Einbürgerungsanreize}

In Abbildung 1 ist dargestellt, welche Vorteile die Befragten mit dem deutschen bzw. türkischen Pass verbinden. Für verschiedene Bereiche wurde die wahrgenommene Instrumentalität der jeweiligen Staatsbürgerschaft auf einer fünfstufigen Skala erhoben. ${ }^{8}$ Die Abbildung weist den Anteil derjenigen aus, die der jeweiligen Nutzendimension „,voll und ganz“ oder „eher“ zugestimmt haben. Dabei wird deutlich, dass auf sämtlichen Nutzendimensionen lediglich der deutsche Pass wirklich hohe Zustimmungsraten erreicht. Ihm wird von $92 \%$ der türkischstämmigen Optionspflichtigen ein hoher rechtlich-pragmatischer Nutzen zugesprochen. In den qualitativen Interviews kommt hier etwa das visumfreie Reisen innerhalb und außerhalb der EU zur Sprache (vgl. auch Weinmann / Becher / Babka von Gostomski 2012: 321ff.). Darüber hinaus sind zwei Drittel der Optionspflichtigen der Ansicht, dass sie mit dem deutschen Pass ihre politischen Interessen besser durchsetzen können.

Abbildung 1: Instrumentalität der deutschen und türkischen Staatsbürgerschaft (Anteile der Befragten mit Zustimmung / voller Zustimmung zu ausgewählten Nützlichkeitsdimensionen)

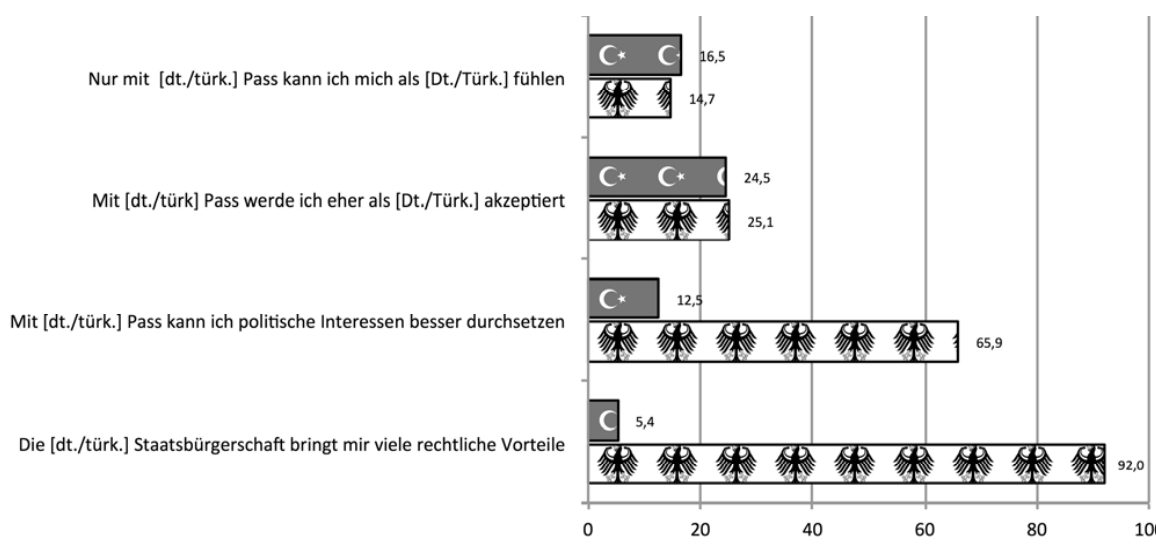

Quelle: Deutsche/r bleiben?

Hinsichtlich des sozialen Nutzens wurde oben schon darauf verwiesen, dass sowohl Eltern als auch Freundinnen und Freunde ganz überwiegend zum deutschen Pass geraten haben bzw. raten. Was den ,außerfamilialen“ sozialen Nutzen des deutschen Passes betrifft zeigt sich, dass ein Viertel der Befragten der Aussage zustimmt, der Pass helfe dabei, als Deutsche bzw. Deutscher akzeptiert zu werden.

Die wahrgenommene Instrumentalität des türkischen Pass beschränkt sich auf dessen Bedeutung für die Akzeptanz als Türkin bzw. Türke. Dies ist der einzige abgefragte Vorteil, der Zustimmungsraten von über $20 \%$ erreicht. Weiterhin stimmen fast $17 \%$ der Aussage zu, sich nur mit einem türkischen Pass auch als Türkin bzw. Türke fühlen zu können. Für eine Minderheit der Optionspflichtigen stellt die Staatsbürgerschaftsentscheidung also durchaus einen Bereich dar, der ihre ethnische Identität bzw. ihr Zugehörigkeitsgefühl berührt. Dies illustriert beispielhaft die Aussage eines befragten Optionspflichtigen:

8 So kann beispielsweise auf die Aussage „Mit einem türkischen Pass werde ich eher als Türke/in akzeptiert“ mit maximaler Zustimmung (1 „Stimme voll und ganz zu“) bis zu äußerster Ablehnung (5 „Stimme überhaupt nicht zu“) reagiert werden. 
Ich würde eine doppelte Staatsbürgerschaft auf Dauer statt einer ,,erzwungenen “ Entscheidung bevorzugen. [...] Jugendliche werden damit gezwungen die Hälfte ihrer Identität aufugeben, obwohl sie sich vielleicht sowohl als Deutscher als auch als Türke fühlen. [Erdal, ${ }^{9}$ 20, Optionsentscheidung noch nicht getroffen, Tendenz zum deutschen Pass, Entscheidung fällt sehr schwer]

Weiterhin stimmen $13 \%$ der Aussage zu, mit einem türkischen Pass ihre politischen Interessen in der Türkei besser umsetzen zu können.

Für die folgenden multivariaten Analysen wurde ein logistisches Regressionsmodell mit einer binär codierten abhängigen Variable geschätzt, die dann eine leichte Entscheidung $(=1)$ anzeigt, wenn den Optionspflichtigen die Staatsangehörigkeitsentscheidung überhaupt nicht schwer fällt und sie sich zudem ihrer Entscheidung sehr sicher sind. Ausgewiesen und grafisch dargestellt werden in Abbildung 2 die durchschnittlichen marginalen Effekte (AME: Average Marginal Effects). Dies entspricht einer allgemeinen Empfehlung und ermöglicht eine leichtere Interpretation der Koeffizienten, da hier der durchschnittliche Einfluss einer unabhängigen Variable auf die Wahrscheinlichkeit mit der das interessierende Ereignis eintritt - hier: eine leichte und sichere Entscheidung - in einer Kennziffer ausgedrückt wird (vgl. Best / Wolf 2012; siehe auch Mood 2009).

Seitens der erklärenden Variablen wurden neben dem Geschlecht und dem Bildungsniveau sowie der subjektiven Diskriminierungswahrnehmung binär codierte Kombinationsvariablen bzw. Interaktionsterme gebildet, denen bei einer hohen individuell wahrgenommenen Instrumentalität des deutschen bzw. türkischen Passes hinsichtlich der jeweiligen Nutzendimension (rechtlich-pragmatisch, sozial, identifikativ) und einer hohen Salienz des entsprechenden Nutzens der Wert 1 zugewiesen wurde. Ein Beispiel: Für die identifikative Nutzendimension des türkischen Passes hat eine Befragte den Wert 1 zugewiesen bekommen, wenn sie einerseits angegeben hat, dass der türkische Pass wichtig ist um sich als Türkin fühlen zu können und sie sich andererseits auch stark als Türkin identifiziert - wodurch die Nutzendimension überhaupt erst salient wird. Entsprechend wurde für den identifikativen Nutzen des deutschen Passes verfahren.

9 Die Namen der Befragten wurden geändert. 
Abbildung 2: Leichtigkeit der Optionsentscheidung (log. Regression: $1=$ Entscheidung sehr sicher und überhaupt nicht schwer; $0=$ Nicht ganz sicher und eher schwer)

Unabhängige Variablen

$$
\begin{array}{r}
\text { Weiblich } \\
\text { (Ref.: männlich) }
\end{array}
$$

Abitur

(Ref.: Geringer Abschluss)

Diskriminierung: sehr oft/oft/manchmal

(Ref.: seltener/nie)

rechtlicher Nutzen (deutscher Pass)*

politischer Nutzen (deutscher Pass)*

politischer Nutzen (türkischer Pass)*

identifikativer Nutzen (deutscher Pass)*

identifikativer Nutzen (türkischer Pass)*

sozialer Nutzen (deutscher Pass)*

sozialer Nutzen (türkischer Pass)*

Vater rät zum türkischen Pass (Ref.: Rat zum dt. Pass/unentschieden)

Mutter rät zum türkischen Pass (Ref.: Rat zum dt. Pass/unentschieden)

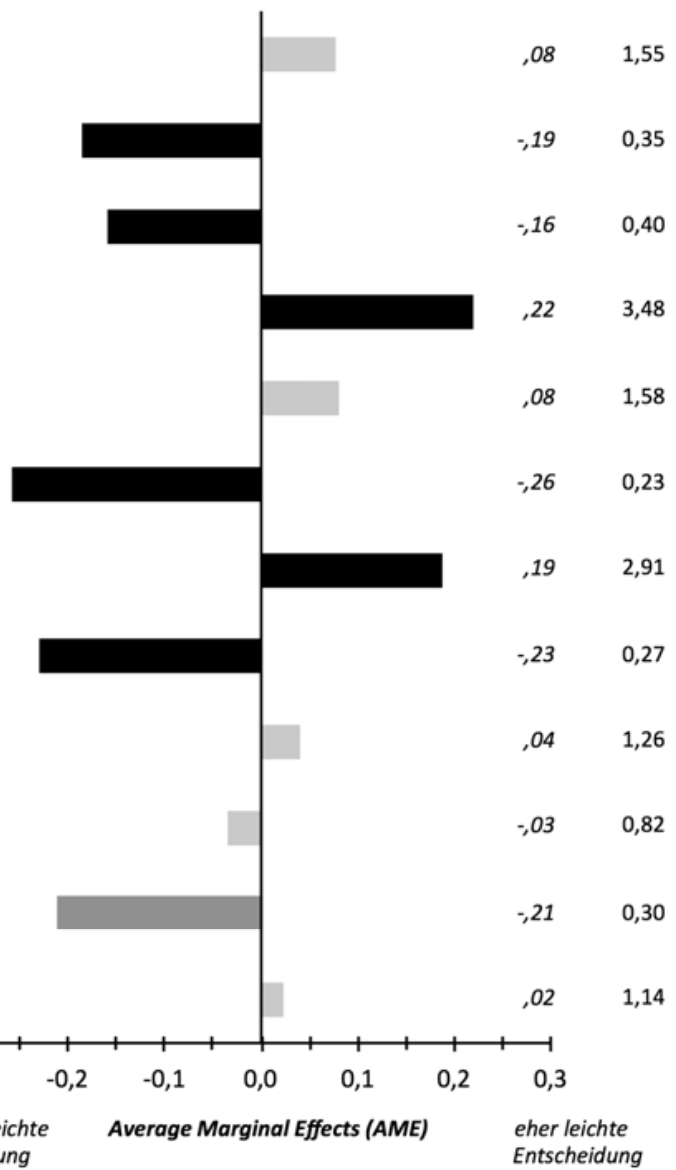

weniger leichte
Entscheidung

Average Marginal Effects (AME)

Entscheidung

\section{$\mathrm{N}=253$}

Pseudo R2=0,240

* Jeweils Interaktionsterme: hohe wahrgenommene Instrumentalität des deutschen / türkischen Passes auf einer Dimension x hohe individuelle Salienz dieser Dimension (Beispiel: identifikativer Nutzen des türkischen Passes $=1$, falls starke Zustimmung zu dem Item „nur mit türkischem Pass kann ich mich als Türke / in fühlen“ und starke Selbstidentifikation als Türke bzw. Türkin).

schwarzer Balken: $\mathrm{p}<, 05$; dunkelgrauer Balken: $\mathrm{p}<, 10$; hellgrauer Balken: $\mathrm{p} \geq, 10$

Quelle: Deutsche/r bleiben?

Für die rechtlich-pragmatische Nutzendimension berücksichtigt das Modell analog dazu den rechtlichen Nutzen des deutschen Passes (wahrgenommene Instrumentalität des rechtlichen 
Nutzens und Bleibeabsicht in Deutschland $)^{10}$ sowie den politischen Nutzen des deutschen und türkischen Passes (wahrgenommene Instrumentalität des deutschen bzw. türkischen Passes für die politische Partizipation und Grad des Interesses an deutscher bzw. türkischer Politik). Hinsichtlich der sozialen Dimension wird ein Interaktionsterm für den sozialen Nutzen des deutschen bzw. türkischen Passes gebildet (wahrgenommene Instrumentalität des deutschen bzw. türkischen Passes für die Akzeptanz als Deutsche/r bzw. Türke/in und ausschließlich nicht-türkischstämmige Freunde bzw. ausschließlich Freunde mit türkischen Wurzeln). Außerdem geht der Rat zur türkischen Staatsbürgerschaft von Vater und Mutter in das Modell mit ein (vgl. Abbildung 2).

Zunächst zeigt sich, dass Frauen gegenüber Männern im Durchschnitt eine um 8 Prozentpunkte (allerdings nicht statistisch signifikante) höhere Wahrscheinlichkeit aufweisen, dass ihnen die Entscheidung leicht fällt. Der negative Effekt für Abiturientinnen und Abiturienten überrascht. Er zeigt an, dass den höher Gebildeten die Optionsentscheidung mit größerer Wahrscheinlichkeit schwer fällt. Dies könnte darauf hindeuten, dass gerade diese Gruppe den von türkischen Optionspflichtigen häufig beklagten „Entscheidungszwang“ des Optionsmodells als Zumutung empfindet (vgl. Worbs / Scholz / Blicke 2012: 147ff.). Sowohl in den qualitativen Interviews als auch in Antworten auf eine offene Frage des quantitativen Instruments kam dies häufiger zur Sprache: „Ich finde es unfair, dass viele andere in Deutschland die doppelte Staatsbürgerschaft legal besitzen dürfen und den Türken das nicht ermöglicht wird“" [Aslan, 20, Optionsentscheidung noch nicht getroffen, Tendenz zum deutschen Pass, Entscheidung fällt überhaupt nicht schwer]. Oder: „Ich finde diese Optionsregelung eigentlich lächerlich, denn ein Land wie Deutschland [...] hat so eine Regelung nicht nötig“ [Cem, 21, optierte für den deutschen Pass, Entscheidung fiel sehr schwer]. Die Entscheidung fällt darüber hinaus deutlich schwerer, wenn die Optionspflichtigen sich in Deutschland diskriminiert fühlen. Dies steht im Einklang zum Paradigma des ethnic boundary crossing. Denn wenn Optionspflichtige die Gefahr sehen, trotz eines deutschen Passes als Ausländer oder Angehörige einer ethnischen Minderheit wahrgenommen oder gar diskriminiert zu werden, dann reduziert dies die Anreize diesen Schritt zu wagen.

Die Ergebnisse bestätigen zunächst die Bedeutung rechtlich-pragmatischer Anreize. Personen, die dem deutschen Pass einen hohen rechtlichen Nutzen zusprechen und für immer in Deutschland bleiben möchten, weisen eine im Durchschnitt um $22 \%$ höhere Wahrscheinlichkeit für eine leichte Optionsentscheidung auf. Auch die Effekte des politischen Interesses weisen in die erwartete Richtung, wenngleich sie für die Türkei nicht zuletzt aufgrund der geringen Fallzahlen keine statistische Signifikanz erreichen: Optionspflichtige, die in der deutschen Staatsbürgerschaft ein wichtiges Instrument zur politischen Partizipation in Deutschland sehen und ein hohes Interesse an dem hiesigen politischen Geschehen berichten, erleben die Optionsentscheidung tendenziell als leichter; analog dazu erschwert ein starkes Interesse an politischer Partizipation in der Türkei die Entscheidung deutlich.

Für die soziale Nutzendimension weisen die Effekte zwar in die erwartete Richtung, sind jedoch statistisch nicht signifikant. Demnach erhöht sich die Wahrscheinlichkeit einer leichteren Entscheidungsfindung für einen Befragten, wenn er der Ansicht ist, dass der deutsche Passes für die Akzeptanz als Deutscher wichtig sei und er zugleich in hohem Maße sozial in die Aufnahmegesellschaft integriert ist und enge Freunde außerhalb der eigenethnischen Gruppe hat. Umgekehrt fällt die Entscheidungsfindung tendenziell schwerer, wenn sämtliche Freunde türkischer Herkunft sind und dem türkischen Pass ein hoher sozialer Nutzen zugeschrieben wird. Mit der Aufgabe des türkischen Passes droht dann möglicherweise ein gewisser

10 Aus modelltechnischen Gründen wird nur der rechtliche Nutzen des deutschen Passes kontrolliert. So ist die Bleibeabsicht in Deutschland mit dem Rückkehrwunsch in die Türkei korreliert, außerdem berichten nur wenige Personen einen hohen rechtlichen Nutzen des türkischen Passes. 
Ansehensverlust innerhalb der eigenethnischen Gruppe. Insgesamt sind diese Effekte jedoch nicht sehr stark ausgeprägt. Hier muss berücksichtigt werden, dass die befragten Optionspflichtigen insgesamt in einem sozialen Umfeld leben, welches dem deutschen Pass sehr positiv gegenübersteht. Zudem hat sich in den qualitativen Interviews gezeigt, dass die Entscheidung oder die Frage der Einbürgerung im Freundeskreis selten thematisiert wird oder zumindest kein konfliktbehaftetes Thema darstellt, Diskussionen hierüber spielen sich eher im Kreis der Familie und mit den Eltern ab (vgl. auch Worbs / Scholz / Blicke 2012). So berichtet eine Interviewte, zwar kurz mit ihrer ebenfalls optionspflichtigen Freundin gesprochen zu haben, ansonsten spiele die Frage der Staatsbürgerschaft aber keine Rolle im Freundeskreis: „Das wird überhaupt gar nicht [...] thematisiert. Unter Freunden oder überhaupt. Wir fragen uns ja nicht gegenseitig: ,Was für einen Pass hast Du bitte?' Ne, [...]" [Sibel, 21, optierte für den deutschen Pass, Entscheidung fiel weniger schwer]. Ein anderer Interviewpartner berichtet zwar durchaus von Gesprächen im Freundeskreis über die Optionsregelung als solche, seine eigene Entscheidung stand dabei jedoch nicht im Fokus: „Ehrlich gesagt, so richtig habe ich darüber gar nicht mehr geredet. Also ich hab mich dann einfach entschieden und es ging einfach so weiter wie es vorher war" [Yilmaz, 22, optierte für den deutschen Pass, Entscheidung fiel weniger schwer]. Interessant ist in diesem Zusammenhang der Befund, dass den Optionspflichtigen die Optionsentscheidung deutlich schwerer fällt, wenn ihnen ihre Väter dazu raten, den türkischen Pass beizubehalten. Der Wunsch bzw. Rat der Mutter ist für die Optionsentscheidung indes unbedeutend. ${ }^{11}$

Schließlich zeigt sich auch für die identifikative Nutzendimension der erwartete Effekt: Denjenigen Befragten, denen der türkische Pass sehr wichtig ist, um sich als Türkin bzw. Türke fühlen zu können und die sich außerdem selbst sehr stark mit der Türkei identifizieren, fällt die Optionsentscheidung schwerer; umgekehrt steigt die Chance für eine leichte Entscheidung bei denjenigen, die im deutschen Pass ein Mittel sehen, sich deutsch fühlen zu können und die sich eher mit den Mehrheitsangehörigen als mit ihrer eigenen ethnischen Gruppe identifizieren. Die Aufgabe des türkischen Passes, dies zeigen auch die geführten qualitativen Interviews immer wieder, wird häufig als emotionales Dilemma beschrieben: „Einerseits fühlt man sich hier heimisch, andererseits ist man ja auch türkisch [...]" [Ayla, 20, Optionsentscheidung noch nicht getroffen, Tendenz zum deutschen Pass, Entscheidung fällt ziemlich schwer]. In diesem Sinne bezeichnen sich einige Optionspflichtige selbst als deutsch mit türkischen Wurzeln. Sie blicken gerade aufgrund ihrer weit fortgeschrittenen strukturellen und kognitiven Integration mit einem besonderen Interesse auf die Türkei. Dies illustriert abschließend die Beschreibung eines jungen Optionspflichtigen, der vor dem Beginn seines Jurastudiums steht, einen Ausbürgerungsantrag gestellt hat und ein starkes Interesse an einer gedeihlichen (politischen) Entwicklung der Türkei aufweist:

[...] (D)amals im Konsulat, da kamen mir auch schon fast die Tränen. [...] Die (türkische Regierung) geht ja wieder in Richtung konservativ. Atatürk war ein sehr moderner Mensch und diesen Teil, hab ich da gedacht, gebe ich jetzt sozusagen auf. Und das hat mich schon sehr traurig gemacht. [Olcay, 21, optierte für den deutschen Pass, Entscheidung fiel ziemlich schwer]

\section{Zusammenfassung und Fazit}

Wir haben in diesem Beitrag die Bestimmungsgründe des Entscheidungsverhaltens türkischstämmiger Optionspflichtiger empirisch untersucht. Bei der Interpretation dieser Ergebnisse gilt es zu beachten, dass die befragten türkischstämmigen jus-soli-Deutschen der Jahrgänge

11 Hier nicht dargestellte Analysen zeigen, dass sich der Wunsch des Vaters auf Beibehaltung des türkischen Passes auf männliche wie weibliche Optionspflichtige gleichermaßen negativ auswirkt und der Rat der Mutter bei beiden Geschlechtern keine Rolle spielt. 
1990 bis 1993 insofern eine selektive Gruppe darstellen, als ihre Eltern die Optionsregelung für ihre Kinder aktiv beantragt haben und offenbar gerade die Familien mit strukturell recht erfolgreich eingegliederten Kindern von dieser Möglichkeit Gebrauch gemacht haben. Dennoch identifiziert sich eine Mehrheit unserer Befragten voll und ganz bzw. überwiegend als Türkin bzw. Türke und nur in mancher Beziehung als Deutsche bzw. Deutscher. Mehr als die Hälfte dieser recht gut gebildeten jungen türkischstämmigen Deutschen ist zudem der Ansicht, dass Türkinnen und Türken in Deutschland aufgrund ihrer Herkunft schlechter behandelt werden, wenngleich „nur“ jede/r Vierte selbst derartige Erfahrungen gemacht hat.

Angesichts des besonderen Zugangswegs dieser Gruppe zur Optionspflicht überrascht zunächst nicht, dass sich eine so klare Mehrheit der hier geborenen und aufgewachsenen „Deutschen auf Zeit" für den deutschen Pass entschieden hat oder beabsichtigt dies zu tun. Dieser Befund deutet auch darauf hin, dass das Einbürgerungsgeschehen außerhalb der Optionsregelung von einem gewissen Trägheitseffekt bestimmt ist. Dieser führt möglicherweise dazu, dass die Einbürgerung angesichts der moderaten rechtlichen Vorteile des deutschen Passes immer weiter aufgeschoben und schließlich fallen gelassen wird. Dieses Trägheitsmoment wird mit der Optionsregelung und dem damit verbundenen Entscheidungszwang außer Kraft gesetzt und zumindest die hier betrachtete Gruppe trifft die Entscheidung dann meist zugunsten des deutschen Passes, wobei sie nicht allen Betroffenen gleichermaßen leicht fällt.

Eine genauere Analyse der Wahrnehmung der deutschen Staatsbürgerschaft zeigt, dass dem deutschen Pass ein hoher rechtlich-pragmatischer Nutzen zugeschrieben wird, weil er beispielsweise das Reisen erleichtert und die volle politische Partizipation ermöglicht. Dem türkischen Pass wird hingegen eher eine sozial-identifikative Bedeutung zugeschrieben, insofern als er in den Augen einer „qualifizierten Minderheit“ der Optionspflichtigen dabei hilft, innerhalb des häufig von Türkischstämmigen dominierten Freundeskreises als Türkin oder Türke akzeptiert zu werden.

Hinsichtlich der Frage, wem die Entscheidung leicht fällt und wem nicht, sprechen unsere Analysen für ein mehrdimensionales Verständnis von Staatsbürgerschaft. Die Entscheidung für oder gegen einen bestimmten Pass ist kein rein pragmatischer Schritt, sondern weist auch ein sozial-identifikatives Moment auf. So wird die Entscheidung für den deutschen Pass dann mit Leichtigkeit und Sicherheit getroffen, wenn man davon überzeugt ist, dass dies Vorteile für das Leben in Deutschland mit sich bringt und eine ohnehin starke Identifikation mit Deutschland verstärkt. Das Gefühl in Deutschland diskriminiert zu werden, eine hohe Bedeutung des türkischen Passes für die Familie und eine starke Identifikation als Türkin bzw. Türke führen demgegenüber dazu, dass sich die Befragten bei der anstehenden Einbürgerungsentscheidung weniger sicher sind und ihnen die Entscheidung schwerer fällt. Die auf der Grundlage des ethnic boundary crossing Paradigmas formulierte Erwartung, dass Kontakte zu Mehrheitsangehörigen die Entscheidung erleichtern, bestätigte sich zwar tendenziell, insgesamt scheint dieser Aspekt jedoch keine zentrale Rolle zu spielen. Vielmehr weisen unsere Ergebnisse darauf hin, dass das innerfamiliäre Einbürgerungsklima für die Entscheidung wichtiger ist als soziale Kontakte außerhalb der Familie. Von großer Bedeutung scheint in diesem Zusammenhang vor allem zu sein, dass der Vater die Einbürgerung seiner Kinder akzeptiert.

Es kann davon ausgegangen werden, dass sich aus dem Pool der „Zauderer“, die sich der Entscheidung nicht sicher sind und diese noch nicht abschließend getroffen haben, vermutlich auch diejenigen rekrutieren werden, die die Entscheidung aufschieben und später qua Behördenentscheid den deutschen Pass verlieren. In Anlehnung an das eingangs zitierte - und in Teilen der Politik durchaus geteilte - Desiderat, dass die Einbürgerung den Endpunkt der Integration darstellen solle, ließe sich jetzt fragen, ob denn dieses Ergebnisse nicht im Einklang mit dem beschriebenen politischen Ziel steht: Diejenigen, die sich noch mit der Türkei identifizieren, später sogar einmal dort wohnen möchten und der deutschen Gesellschaft insofern 
mit Skepsis begegnen, als sie diese als diskriminierend wahrnehmen, wären eben noch nicht weitgehend genug integriert, um ,ganz und gar Deutsch“ sein zu wollen - und zu sollen. Gegen diese Interpretation spricht, dass es gerade auch die jungen Erwachsenen mit höherer Bildung sind, denen die Optionsentscheidung schwer fällt. Offenbar fühlen sich durch das deutsche Festhalten an dem Prinzip der Vermeidung von Mehrstaatigkeit, das im Entscheidungsdruck der Optionsregelung zum Ausdruck kommt, gerade die strukturell gut integrierten jungen Migrantinnen und Migranten der zweiten Generation gegängelt. Der Befund, dass ausgerechnet diese Gruppe mit der Aufgabe des türkischen Passes „ein Problem hat“ mag manch einer als Zeichen der Entwarnung interpretieren. Schließlich passt er so gar nicht zu dem Bild einer gescheiterten und verweigerten Integration. Wenn man aber davon ausgeht, dass die türkischen Abiturientinnen und Abiturienten der zweiten Generation von heute die Hochqualifizierten von morgen sind, dann dürfte zumindest diese Folge der Optionsregelung kaum im Sinne ihrer Erfinder sein.

\section{Literatur}

Aguirre, B. / R. Saenz (2002): Testing the Effects of Collectively Expected Durations of Migration: The Naturalization of Mexicans and Cubans, in: International Migration Review 36, S. 102-124.

Alba, R. (2005): Bright vs. Blurred Boundaries. Second-Generation Assimilation and Exclusion in France, Germany, and the United States, in: Ethnic and Racial Studies 28, S. 20-49.

Alvarez, R. (1987): A Profile of the Citizenship Process among Hispanics in the United States, in: International Migration Review 21, S. 327-351.

American Association for Public Opinion Research (AAPOR) (2011): Standard Definitions, Lenexa / KS.

Barkan, E. / N. Khokhlov (1980): Socioeconomic Data as Indices of Naturalization Patterns in the United States: A Theory Revisited, in: Ethnicity 7, S. 159-190.

Bernard, W. (1936): Cultural Determinants of Naturalization, in: American Sociological Review 1, S. 943-953.

Best, H. / C. Wolf(2012): Modellvergleich und Ergebnisinterpretation in Logit- und Probit-Regressionen, in: Kölner Zeitschrift für Soziologie und Sozialpsychologie 64, S. 377-395.

Bloemraad, I. (2006): Becoming a Citizen in the United States and Canada: Structured Mobilization and Immigrant Political Incorporation, in: Social Forces 85, S. 667-695.

Borjas, G. (2001): Welfare Reform and Immigration, in: R. Blank / R. Haskins (Hrsg.), The New World of Welfare. Washington, D.C., S. 369-385.

BVerfG (21.5.1974): 1 BvL 22 / 71 u. $21 / 72$.

Castles, S. (1994): Democracy and Multicultural Citizenship. Australian Debates and their Relevance for Western Europe, in: R. Bauböck (Hrsg.), From Aliens to Citizens. Redefining the Status of Immigrants in Europe, Aldershot, S. 3-27.

Deutscher Bundesrat (2001): Plenarprotokoll 763, Berlin.

Deutscher Bundestag (2008): Drucksache 16 / 8092. Antwort der Bundesregierung auf die Kleine Anfrage „Auswirkungen der staatsangehörigkeitsrechtlichen Optionsregelung“, Berlin.

Deutscher Bundestag (2011): Plenarprotokoll 17 / 139, Berlin.

Diehl, C. / M. Blohm (2003): Rights or Identity? Naturalization Processes among „Labor Migrants“ in Germany, in: International Migration Review 37, S. 133-162.

Diehl, C. / M. Blohm (2008): Die Entscheidung zur Einbürgerung. Optionen, Anreize und identifikative Aspekte, in: F. Kalter (Hrsg.), Migration und Integration. Kölner Zeitschrift für Soziologie und Sozialpsychologie, Sonderheft 48, Wiesbaden, S. 437-464. 
Diehl, C. / M. Blohm (2011): Naturalization as Boundary Crossing: Evidence from Labor Migrants in Germany, in: A. Azzi / X. Chryssochoou / B. Klandermans / B. Simon (Hrsg.), Identity and Participation in Culturally Diverse Societies. A Multidisciplinary Perspective, Malden / MA, S. 317-337.

Dornis, C. (2002): Zwei Jahre nach der Reform des Staatsangehörigkeitsrechts. Bilanz und Ausblick, in: K. Bade / R. Münz (Hrsg.), Migrationsreport 2002, Frankfurt / Main, S. 163-177.

Euwals, R. / J. Dagevos / M. Gijsberts / H. Roodenburg (2010): Citizenship and Labor Market Position: Turkish Immigrants in Germany and the Netherlands, in: International Migration Review 44, S. $513-538$.

Evans, M. (1988): Choosing to Be a Citizen: The Time-Path of Citizenship in Australia, in: International Migration Review 22, S. 243-264.

Fick, P. / C. Diehl (2013): Incentivierungsstrategien bei Minderheitenangehörigen. Ergebnisse eines Methodenexperiments (eingereichtes Manuskript).

Freeman, G. / L. Plascencia / S. Baker / M. Orozco (2002): Explaining the Surge in Citizenship Applications in the 1990 s: Lawful Permanent Residents in Texas, in: Social Science Quarterly 83, S. 1013-1025.

Gerdes, J. / T. Faist (2006): Von ethnischer zu republikanischer Integration. Der Diskurs um die Reform des deutschen Staatsangehörigkeitsrechts, in: Berliner Journal für Soziologie 16, S. 313-335.

Goebel, J. / C. Spieß / N. Witte / S. Gerstenberg (2007): Die Verknüpfung des SOEP mit MICROMIndikatoren: Der MICROM-SOEP Datensatz. Data Documentation 26, Berlin.

Goldberg, A. / F. Sen (Hrsg.) (1999): Deutsche Türken - Türkische Deutsche? Die Diskussion um die doppelte Staatsbürgerschaft, Münster.

Gresch, C. / C. Kristen (2011): Staatsbürgerschaft oder Migrationshintergrund? Ein Vergleich unterschiedlicher Operationalisierungsweisen am Beispiel der Bildungsbeteiligung, in: Zeitschrift für Soziologie 40, S. 208-227.

Göbel-Zimmermann, R. (2004): Das neue Staatsangehörigkeitsrecht. Erfahrungen und Reformvorschläge, in: K. Barwig / U. Davy (Hrsg.), Auf dem Weg zur Rechtsgleichheit? Konzepte und Grenzen einer Politik der Integration von Einwanderern, Baden-Baden, S. 148-168.

Hagedorn, H. (2001): Wer darf Mitglied werden? Einbürgerung in Deutschland und Frankreich im Vergleich, Opladen.

Hammar, T. (1990): Democracy and the Nation State. Aliens, Denizens and Citizens in a World of International Migration, Aldershot.

Hochman, O. (2011): Determinants of Positive Naturalisation Intentions among Germany's Labour Migrants, in: Journal of Ethnic and Migration Studies 37, S. 1403-1421.

Van Hook, J. / S. Brown / F. Bean (2006): For Love or Money? Welfare Reform and Immigrant Naturalization, in: Social Forces 85, S. 643-666.

Jasso, G. / M. Rosenzweig (1986): Family Reunification and the Family Multiplier: U.S. Immigration Law, Origin-Country Conditions, and the Reproduction of Immigrants, in: Demography 23, S. 291-311.

Joppke, C. (2007): Transformation of Citizenship: Status, Rights, Identity, in: Citizenship Studies 11, S. 37-48.

Joppke, C. (2010 a): Citizenship and Immigration, Cambridge.

Joppke, C. (2010 b): The Inevitable Lightening of Citizenship, in: European Journal of Sociology 51, S. 9-32.

Liang, Z. (1994): Social Contact, Social Capital, and the Naturalization Process: Evidence from Six Immigrant Groups, in: Social Science Research 23, S. 407-437.

Lämmermann, F. (2011): Ein Jahrzehnt ius soli - Bilanz und Ausblick, in: Zeitschrift für Ausländerrecht und Ausländerpolitik 31, S. 1-8.

Marshall, T. (1950): Citizenship and Social Class, in: T. Marshall (Hrsg.), Class, Citizenship, and Social Development. New York / NY, S. 65-122. 
Massey, D. / I. Akresh (2006): Immigrant Intentions and Mobility in a Global Economy: The Attitudes and Behavior of Recently Arrived U.S. Immigrants, in: Social Science Quarterly 87, S. 954-971.

Mertens, K. (2004): Das neue deutsche Staatsangehörigkeitsrecht - eine verfassungsrechtliche Untersuchung, Berlin.

Mood, C. (2009): Logistic Regression: Why We Cannot Do What We Think We Can Do, and What We Can Do About It, in: European Sociological Review 26, S. 67-82.

v. Münch, I. (2007): Die deutsche Staatsangehörigkeit. Vergangenheit - Gegenwart - Zukunft, Berlin.

Nassehi, A. (2010): Citizenship and Modernity, in: T. K. Oomen (Hrsg.), Classes, Citizenship and Inequality, New Delhi, S. 186-208.

Pantoja, A. / S. Gershon (2006): Political Orientations and Naturalization among Latino and Latina Immigrants, in: Social Science Quarterly 87, S. 1171-1187.

Plascencia, L. / G. Freeman / M. Setzler (2003): The Decline of Barriers to Immigrant Economic and Political Rights in the American States: 1977-2001, in: International Migration Review 37, S. 5-23.

Portes, A. / J. Curtis (1987): Changing Flags: Naturalization and its Determinants Among Mexican Immigrants, in: International Migration Review 21, S. 352-371.

Portes, A. / R. Mozo (1985): The Political Adaption Process of Cubans and Other Ethnic Minorities in the United States: A Preliminary Analysis, in: International Migration Review 19, S. 35-63.

Portes, A. / R. Rumbaut (1996): Immigrant America. A Portrait, Berkeley / CA.

Prümm, K. (2004): Einbürgerung als Option. Die Bedeutung des Wechsels der Staatsangehörigkeit für Menschen türkischer Herkunft in Deutschland, Münster.

Santel, B. (1998): Auf dem Weg zur Konvergenz? Einwanderungspolitik in Deutschland und den Vereinigten Staaten im Vergleich, in: Zeitschrift für Ausländerrecht und Ausländerpolitik 8, S. 14-20.

Schiffauer, W. (2007): Der unheimliche Muslim - Staatsbürgerschaft und zivilgesellschaftliche Ängste, in: M. Wohlrab-Sahr / L. Tezcan (Hrsg.), Konfliktfeld Islam in Europa. Soziale Welt Sonderband 17, Baden-Baden, S. 111-134.

Schiffauer, W. (2008): Parallelgesellschaften. Wie viel Wertekonsens braucht unsere Gesellschaft? Für eine kluge Politik der Differenz, Bielefeld.

Schuck, P. (1998): Citizens, Strangers, and In-Betweens. Essays on Immigration and Citizenship, Boulder/ CO.

Statistisches Bundesamt (2012): Fachserie 1, Reihe 2.1, Wiesbaden.

Weil, P. (2001): Zugang zur Staatsbürgerschaft. Ein Vergleich von 25 Staatsangehörigkeitsgesetzen, in: C. v. Christoph / J. Kocka (Hrsg.), Staatsbürgerschaft in Europa. Historische Erfahrungen und aktuelle Debatten, Hamburg, S. 92-111.

Weinmann, M. / I. Becher / C. Babka von Gostomski (2012): Einbürgerungsverhalten von Ausländerinnen und Ausländern in Deutschland sowie Erkenntnisse zu Optionspflichtigen, Nürnberg: BAMF.

Wimmer, A. (2008): Elementary Strategies of Ethnic Boundary Making, in: Ethnic and Racial Studies 31, S. 1025-1055.

Wobbe, T. / R. Otte (2000): Politische Institutionen im gesellschaftlichen Wandel. Einbürgerung in Deutschland zwischen Erwartungen von Migranten und staatlicher Vorgabe, in: Zeitschrift für Soziologie 29, S. 444-462.

Worbs, S. / A. Scholz / S. Blicke (2012): Die Optionsregelung im Staatsangehörigkeitsrecht aus der Sicht von Betroffenen, Nürnberg: BAMF.

Yang, P. (1994 a): Ethnicity and Naturalization, in: Ethnic and Racial Studies 17, S. 593-618.

Yang, P. (1994 b): Explaining Immigrant Naturalization, in: International Migration Review 28, S. 449-477. 
Prof. Dr. Claudia Diehl Patrick Fick

Universität Göttingen Institut für Soziologie

Platz der Göttinger Sieben 3 37073 Göttingen cdiehl@uni-goettingen.de patrick.fick@sowi.uni-goettingen.de 